\title{
Optimal control of a fractional order epidemic model with application to human respiratory syncytial virus infection*
}

\author{
Silvério Rosa ${ }^{a}$ \\ rosa@ubi.pt \\ Delfim F. M. Torres ${ }^{b \dagger}$ \\ delfim@ua.pt \\ ${ }^{a}$ Instituto de Telecomunicações and Department of Mathematics, \\ Universidade da Beira Interior, 6201-001 Covilhã, Portugal \\ ${ }^{b}$ Center for Research and Development in Mathematics and Applications \\ (CIDMA), Department of Mathematics, \\ University of Aveiro, 3810-193 Aveiro, Portugal
}

\begin{abstract}
A human respiratory syncytial virus surveillance system was implemented in Florida in 1999, to support clinical decision-making for prophylaxis of premature newborns. Recently, a local periodic SEIRS mathematical model was proposed in [Stat. Optim. Inf. Comput. 6 (2018), no. 1, 139-149] to describe real data collected by Florida's system. In contrast, here we propose a non-local fractional (non-integer) order model. A fractional optimal control problem is then formulated and solved, having treatment as the control. Finally, a cost-effectiveness analysis is carried out to evaluate the cost and the effectiveness of proposed control measures during the intervention period, showing the superiority of obtained results with respect to previous ones.
\end{abstract}

Keywords: Human respiratory syncytial virus (HRSV); compartmental mathematical models; fractional optimal control.

MSC 2010: 34A08, 49M05, 92D30.

\section{Introduction}

Human respiratory syncytial virus (HRSV) is a virus that causes respiratory tract infections. It is a major cause of lower respiratory tract infections and hospital visits during infancy and childhood. A prophylactic medication, palivizumab, can be employed to prevent HRSV in pre-term infants (under 35 weeks gestation), infants with certain congenital heart defects or bronchopulmonary dysplasia, and infants with congenital malformations of the airway. Treatment is limited to supportive care, including oxygen therapy. In temperate climates, there is an annual epidemic during the winter season. In tropical climates, infection is most common during the rainy season. In the United States, $60 \%$ of infants are infected during their first HRSV season, and nearly all children will have been infected with the virus by two to three years of age [22]. Of those infected with HRSV, 2 to $3 \%$ will develop bronchiolitis, necessitating hospitalization [23. Natural infection with HRSV induces protective immunity, which wanes over time, possibly more so than other respiratory viral infections, and thus people can be infected multiple times. Sometimes, an infant can become symptomatically infected more than once, even within a single HRSV season.

${ }^{*}$ This is a preprint of a paper whose final and definite form is with Chaos, Solitons $\&$ Fractals, available from http://www.elsevier.com/locate/issn/09600779 Submitted 23-July-2018; Revised 14-Oct-2018; Accepted 15Oct-2018.

${ }^{\dagger}$ Corresponding author. 
Severe HRSV infections have increasingly been found among elderly patients. Young adults can be re-infected every five to seven years, with symptoms looking like a sinus infection or a cold. The Florida Department of Health provides an integrated and reliable HRSV system, with data from hospitals and laboratories [21.

Mathematical models can project how infectious diseases progress, to show the likely outcome of an epidemic, and help inform public health interventions. In epidemiology, compartmental models serve as the base mathematical framework for understanding the complex dynamics of these systems. Such compartments, in the simplest case, stratify the population into two health states: susceptible to the infection of the pathogen, often denoted by $S$, and infected by the pathogen, often denoted by the symbol $I$. The way that these compartments interact is based upon phenomenological assumptions, and the model is built up from there. These models are usually investigated through ordinary differential equations. Depending on the disease, other compartments may be included, most notably the recovered/removed/immune compartment, often denoted by $R$. Recently, to push such models to further realism and taking into account the influence of past on the current and future state of the diseases, they have been characterized mathematically with the help of fractional order differential equations: see, e.g., investigations in dengue [32, Ebola [10, tuberculosis [42] and HIV/AIDS [16, 43.

A crucial question consists to find parameters for the particular disease under study, and use those parameters to calculate the effects of possible control interventions, like treatment or vaccination. Then the central issue is how to implement such interventions in an optimal way. This investigation program has been carried out for several infectious diseases with classical integerorder compartmental models: see, e.g., [36 for HRSV and [29] and [11, 33, 34] for Zika and Ebola viruses, respectively, where the implementation of optimal control interventions are proposed. Here we extend such approach with new fractional compartmental models. Applications of fractional calculus in numerous fields of science and engineering have gained popularity and importance in past four decades, see [3, 37, 38] and references therein. Recently, extensions of fractional derivative operators have been developed and proved to be very useful in several applications, showing a high vitality of the research field [5, 6, 17. An extension of the Caputo fractional derivative operator is given in [26] by using a generalized beta function, Saigo-Maeda fractional differential operators involving Appell functions are investigated in [2, while fractional integral operators involving Gauss hypergeometric functions are studied in [18. For variable-order fractional operators see [9] and references therein. Fractional operators on arbitrary time scales are proposed and investigated in [14, 15]. Usually, numerical techniques for solving such fractional order models are required 4, 40. To the best of our knowledge, this is the first work to use fractional calculus and fractional optimal control in the study of HRSV. For that we use derivatives in the standard Caputo sense.

A comparison of the standard SIRS model with a more complex integer-order model of HRSV transmission, in which individuals acquire immunity gradually after repeated exposure to infection, is given in [41. In [1], an age-structured mathematical model for HRSV is proposed, where children younger than one year old, who are the most affected by this illness, are specially considered. A numerical scheme for the SIRS seasonal epidemiological model of HRSV transmission is proposed in [13]. It turns out that solutions for HRSV compartmental models are typically memory-periodic systems [12]. For this reason, in this work we propose the use of fractional optimal control theory [19, 24 to a non-autonomous fractional SEIRS model, and show its usefulness according with real HRSV data provided by the Florida Department of Health [21.

The paper is organized as follows. In Section 2, we introduce the SIRS- $\alpha$ and SEIRS- $\alpha$ fractional epidemic models, which generalize corresponding integer-order $(\alpha=1)$ models of [28]. The main results are then given in Section 3 , estimation of the fractional order $\alpha$ with real data of Florida, for the two proposed fractional models (Section 3.1); fractional optimal control, costeffectiveness and numerical simulations for the more realistic SEIRS- $\alpha$ model (Sections 3.2 and 3.3). We end with Section 4 of conclusions and perspectives of future work. 


\section{Fractional compartmental models}

We focus on compartmental models that divide the population into mutually exclusive distinct groups (of susceptible, or infected, or immune individuals, or ...) and we use deterministic continuous transitions between those groups, also known as states. Due to the seasonality of HRSV, the models that best fit real data are periodic. In [41, two integer-order models are proposed, where the transmission is periodic: (i) a simple model with only three compartments, known as SIRS, which we extend here to the fractional SIRS- $\alpha$ case in Section 2.1; (ii) and a more complex model with seventeen compartments, named MSEIRS4. However, it is shown that the simpler SIRS model fits better real data [41. Zang et al. 44] use a non-autonomous SEIR model where, beyond the periodicity in the transmission rate, the annual recruitment rate is also periodic. This assumption is due to opening and closing of schools. Here we generalize such ideas and consider a simple periodic fractional SEIRS- $\alpha$ model (Section 2.2).

\section{$2.1 \quad$ SIRS- $\alpha$ model}

In our first fractional model, we consider that the population consists of susceptible $(S)$, infected and infectious $(I)$, and recovered $(R)$ individuals. A characteristic feature of HRSV is that immunity after infection is temporary, so that the recovered individuals become susceptible again [41]. Let parameter $\mu$ denote the birth rate, which we assume equal to the mortality rate; $\gamma$ be the rate of loss of immunity; and $\nu$ the rate of loss of infectiousness. The influence of the seasonality on the transmission parameter $\beta$ is modeled by the cosine function. Using a linear mass fractional action law, we propose the following system of fractional differential equations:

$$
\left\{\begin{array}{l}
{ }_{0}^{C} D_{t}^{\alpha} S(t)=\mu-\mu S(t)-\beta(t) S(t) I(t)+\gamma R(t), \\
{ }_{0}^{C} D_{t}^{\alpha} I(t)=\beta(t) S(t) I(t)-\nu I(t)-\mu I(t), \\
{ }_{0}^{C} D_{t}^{\alpha} R(t)=\nu I(t)-\mu R(t)-\gamma R(t),
\end{array}\right.
$$

where $\beta(t)=b_{0}\left(1+b_{1} \cos (2 \pi t+\Phi)\right)$ and ${ }_{0}^{C} D_{t}^{\alpha}$ denotes the left Caputo derivative of order $\alpha \in(0,1]$ [31, 39]. The parameter $b_{0}$ is the mean of the transmission parameter and $b_{1}$ is the amplitude of the seasonal fluctuation in the transmission parameter, $\beta$.

Remark 1. When $\alpha=1$, the fractional compartmental model (11) represent the classical SIRS model investigated in 28.

We improve previous paper [36], where two models were used: an SIRS and a SEIRS model. The first model, periodic in the transmission rate, is the simplest, however, as already mentioned, it proven to be better than a MSEIRS4 model, which is a more complex model. The second model, adds to the former periodicity in the recruitment rate, fitting better the real data [36]. Here we investigate SIRS and SEIRS like models, denoted by SIRS- $\alpha$ (1) and SEIRS- $\alpha$ (2), where $\alpha$ denotes the non-integer order of differentiation under consideration, that are better than the (integer-order) models investigated in [36]. This makes our new $\alpha$-models interesting from a mathematical modeling point of view.

\section{$2.2 \quad$ SEIRS- $\alpha$ model}

To incorporate more features of HRSV, we extend the previous fractional-order model in the following way. First, we include a latency period by introducing a group $E$ of individuals who have been infected but are not yet infectious. These individuals become infectious at a rate $\varepsilon$. We assume the latency period to be equal to the time between infection and the first symptoms. As in [44, we consider that the annual recruitment rate is seasonal due to schools opening/closing 
periods. Our system of fractional differential equations is now given by

$$
\left\{\begin{array}{l}
{ }_{0}^{C} D_{t}^{\alpha} S(t)=\lambda(t)-\mu S(t)-\beta(t) S(t) I(t)+\gamma R(t), \\
{ }_{0}^{C} D_{t}^{\alpha} E(t)=\beta(t) S(t) I(t)-\mu E(t)-\varepsilon E(t), \\
{ }_{0}^{C} D_{t}^{\alpha} I(t)=\varepsilon E(t)-\mu I(t)-\nu I(t), \\
{ }_{0}^{C} D_{t}^{\alpha} R(t)=\nu I(t)-\mu R(t)-\gamma R(t),
\end{array}\right.
$$

where $\lambda(t)=\mu\left(1+c_{1} \cos (2 \pi t+\Phi)\right)$ is the recruitment rate (including newborns and immigrants), parameter $c_{1}$ is the amplitude of the seasonal fluctuation in the recruitment parameter, $\lambda$, while $\Phi$ is an angle that is chosen in agreement with real data, and, as before, ${ }_{0}^{C} D_{t}^{\alpha}$ denotes the left Caputo derivative of order $\alpha \in(0,1]$. Note that in the particular case $\alpha=1$ we obtain from (2) the SEIRS model of [28].

\section{Main results}

We begin by investigating how realistic the fractional models discussed in Section 2 are, with respect to HRSV and real data from Florida 21. For that, we borrow the values for the parameters $\mu, \nu, \gamma, \varepsilon, b_{0}, b_{1}, c_{1}$, and $\Phi$ from [36] and do a proper estimation of the best fractional order $\alpha$ for models SIRS- $\alpha$ and SEIRS- $\alpha$.

\subsection{Estimation of the fractional order $\alpha$ for Caputo derivatives}

Using the parameters values as given in Table 1, we searched the fractional order of differentiation, $\alpha$, that best fits the data on the reported number of positive tests of HRSV disease, per month, in the state of Florida, excluding North region, between September 2011 and July 2014 (35 months). The data was obtained from the Florida Department of Health [21. The value of the derivative order, $\alpha$, was obtained by a search on the interval ]0,1]. We started with $\alpha=1$ and successively lower its value until we find one whose lower values in the neighborhood correspond to worst fitting results. The results for the SIRS- $\alpha$ model (1) are given in Figure 1 while the results corresponding to the SEIRS- $\alpha$ model (2) are given in Figure 2 ,

Table 1: Models' parameters borrowed from [36].

\begin{tabular}{ccccccccc}
\hline model & $\mu$ & $\nu$ & $\gamma$ & $\varepsilon$ & $b_{0}$ & $b_{1}$ & $c_{1}$ & $\Phi$ \\
\hline SIRS- $\alpha$ & 0.0113 & 36 & 1.8 & - & 74.2 & 0.14 & - & $7 \pi / 5$ \\
SEIRS- $\alpha$ & 0.0113 & 36 & 1.8 & 91 & 88.25 & 0.17 & 0.17 & $7 \pi / 5$ \\
\hline
\end{tabular}

Table 2: Comparison of models (11) and (2) with real data. The $l_{2}$ norm of the difference between real data and the predictive cases given by the models is denoted by error. In the last column, relative error denotes the percentual difference of infants, per year, with respect to the total child population of Florida in 2014.

\begin{tabular}{llcc}
\hline model & $\alpha$ & error & relative error $(\%)$ \\
\hline \multirow{2}{*}{ SIRS- $\alpha$} & 1.0 & 1871.46 & 0.06567 \\
& 0.968 & 1840.90 & 0.06460 \\
\hline \multirow{2}{*}{ SEIRS- $\alpha$} & 1.0 & 1719.12 & 0.06032 \\
& 0.993 & 1716.91 & 0.06025 \\
\hline
\end{tabular}




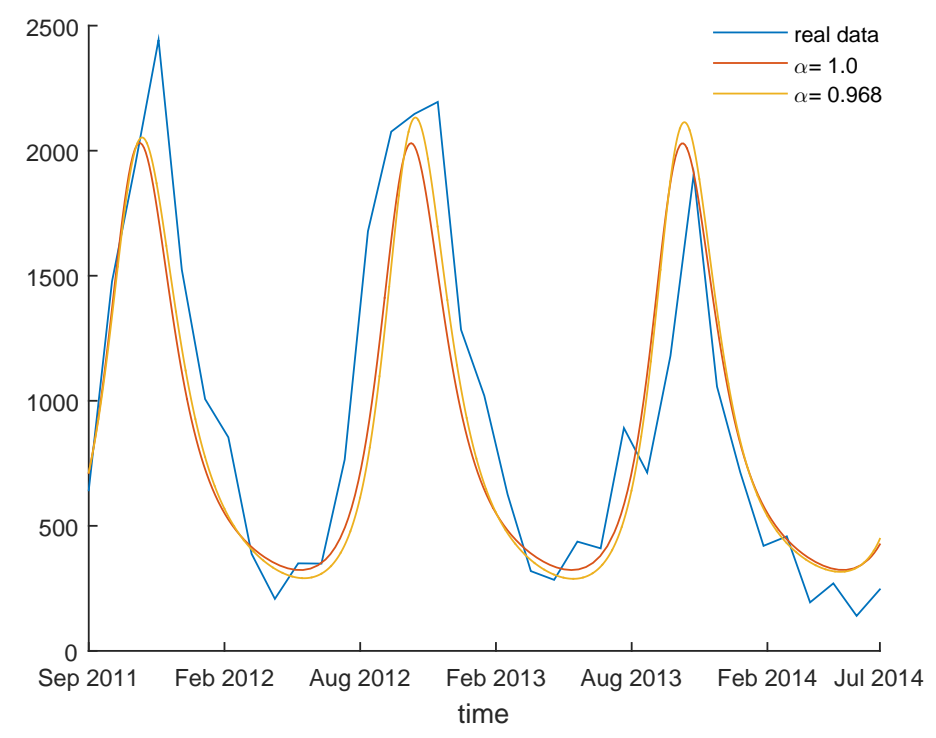

Figure 1: Comparison of infected/infectious individuals $I(t)$ : real data, classical SIRS model (i.e., $\alpha=1)$ and the fractional SIRS- $\alpha$ model with $\alpha=0.968$.

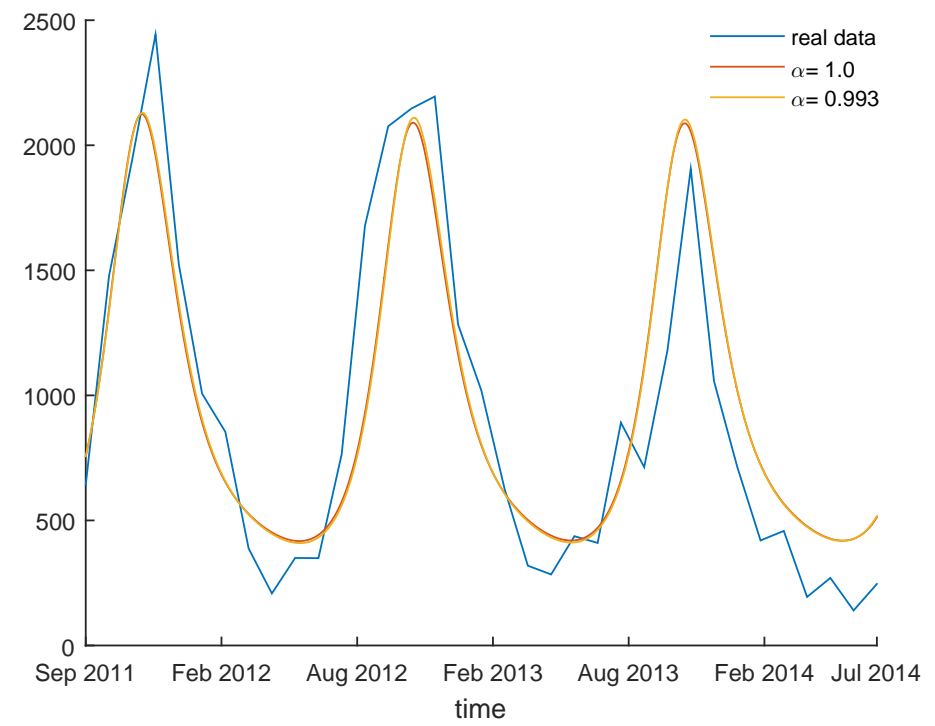

Figure 2: Comparison of infected/infectious individuals $I(t)$ : real data, classical SEIRS model (i.e., $\alpha=1$ ) and the fractional SEIRS- $\alpha$ model with $\alpha=0.993$.

For comparison reasons, we adopted the same fitting approach as in 36, which consists in the minimization of the $l_{2}$ norm of the difference between real data and the number of HRSV infection individuals predicted by the models. Table 2 gives the values of the errors for the considered models. The relative errors show that these models fit quite well the data of HRSV disease. Comparing the results for the optimal values of $\alpha$, that is, $\alpha=0.968$ for the fractional order model (1) and $\alpha=0.993$ for the fractional order model (2), with the classical SIRS and SEIRS models obtained for $\alpha=1$, one concludes that while the absolute error of the fractional SIRS- $\alpha$ model reduces more than the homonymous error of the fractional SEIRS- $\alpha$ model, it is 
the SEIRS- $\alpha$ model the one with lowest error.

\subsection{Fractional optimal control problem}

In order to investigate some optimal measures, we choose, in agreement with Section 3.1, the SEIRS- $\alpha$ model with $\alpha=0.993$, which is the one that provides the best fitting to the considered real data. The evolution of the variables of the model depend on some circumstances that can be controlled. In what concerns HRSV disease, treatment is the most commonly used. Hence, we consider the following fractional optimal control problem: to minimize the number of infectious individuals and the cost associated to control the disease with the treatment of the patients, that is,

$$
\min \mathcal{J}(I, \mathbb{T})=\int_{0}^{t_{f}}\left(\kappa_{1} I(t)+\kappa_{2} \mathbb{T}^{2}(t)\right) d t
$$

with given $0<\kappa_{1}, \kappa_{2}<\infty$, subject to the fractional control system

$$
\left\{\begin{array}{l}
{ }_{0}^{C} D_{t}^{\alpha} S(t)=\lambda(t)-\mu S(t)-\beta(t) S(t) I(t)+\gamma R(t), \\
{ }_{0}^{C} D_{t}^{\alpha} E(t)=\beta(t) S(t) I(t)-\mu E(t)-\varepsilon E(t), \\
{ }_{0}^{C} D_{t}^{\alpha} I(t)=\varepsilon E(t)-\mu I(t)-\nu I(t)-\mathbb{T}(t) I(t), \\
{ }_{0}^{C} D_{t}^{\alpha} R(t)=\nu I(t)-\mu R(t)-\gamma R(t)+\mathbb{T}(t) I(t)
\end{array}\right.
$$

and given initial conditions

$$
S(0), E(0), I(0), R(0) \geqslant 0 .
$$

Here, $\mathbb{T}$ is the control variable, which designates treatment. Note that in absence of treatment, that is, for $\mathbb{T}(t) \equiv 0$, then the control system (4) reduces to the SEIRS- $\alpha$ dynamical system (2). The set of admissible control functions is

$$
\Omega=\left\{\mathbb{T}(\cdot) \in L^{\infty}\left(0, t_{f}\right): 0 \leqslant \mathbb{T}(t) \leqslant \mathbb{T}_{\max }, \forall t \in\left[0, t_{f}\right]\right\}
$$

Pontryagin's maximum principle (PMP) for fractional optimal control can be used to solve the problem [7, 8, 25, 27. The Hamiltonian of our optimal control problem is

$$
\begin{aligned}
\mathcal{H}=\kappa_{1} I+\kappa_{2} \mathbb{T}^{2}+p_{1}(\lambda-\mu S-\beta S I+ & \gamma R)+p_{2}(\beta S I-\mu E-\varepsilon E) \\
& +p_{3}(\varepsilon E-\mu I-\nu I-\mathbb{T} I)+p_{4}(\nu I-\mu R-\gamma R+\mathbb{T} I)
\end{aligned}
$$

the optimality condition of PMP ensures that the optimal control is given by

$$
\mathbb{T}(t)=\min \left\{\max \left\{0, \frac{\left(p_{3}(t)-p_{4}(t)\right) I(t)}{2 \kappa_{2}}\right\}, \mathbb{T}_{\max }\right\} ;
$$

while the adjoint system asserts that the co-state variables $p_{i}(t), i=1, \ldots, 4$, satisfy

$$
\left\{\begin{aligned}
{ }_{t} D_{t_{f}}^{\alpha} p_{1}(t) & =p_{1}(t)(\mu+\beta(t) I(t))-\beta(t) I(t) p_{2}(t), \\
{ }_{t} D_{t_{f}}^{\alpha} p_{2}(t) & =p_{2}(t)(\mu+\varepsilon)-\varepsilon p_{3}(t) \\
{ }_{t} D_{t_{f}}^{\alpha} p_{3}(t)= & -\kappa_{1}+\beta(t) p_{1}(t) S(t)-p_{2}(t) \beta(t) S(t) \\
& +p_{3}(t)(\mu+\nu+\mathbb{T}(t))-p_{4}(t)(\nu+\mathbb{T}(t)) \\
{ }_{t} D_{t_{f}}^{\alpha} p_{4}(t)= & -\gamma p_{1}(t)+p_{4}(t)(\mu+\gamma)
\end{aligned}\right.
$$

which is a fractional system of right Riemann-Liouville derivatives ${ }_{t} D_{t_{f}}^{\alpha}$. In addition, the following transversality conditions hold:

$$
\left.{ }_{t} D_{t_{f}}^{\alpha-1} p_{i}\right|_{t_{f}}=\left.0 \Leftrightarrow{ }_{t} I_{t_{f}}^{1-\alpha} p_{i}\right|_{t_{f}}=p_{i}\left(t_{f}\right)=0, \quad i=1, \ldots, 4
$$

where ${ }_{t} I_{t_{f}}^{1-\alpha}$ is the right Riemann-Liouville fractional integral of order $1-\alpha$. 


\subsection{Numerical results and cost-effectiveness analysis of the fractional HRSV optimal control problem}

The optimal control problem (3) -(6) is numerically solved with the help of PMP and its optimality conditions, as discussed in Section 3.2, in the classical $(\alpha=1)$ and fractional ( $\alpha=0.993)$ cases, using the predict-evaluate-correct-evaluate (PECE) method of Adams-Basforth-Moulton [20]. First we solve system (4) by the PECE procedure with initial conditions for the state variables (5) given in terms of percentage of total population, that is, $S(0)+E(0)+I(0)+R(0)=1$, and a guess for the control over the time interval $\left[0, t_{f}\right]$, and obtain the values of the state variables $S, E, I$ and $R$. Applying the change of variable

$$
t^{\prime}=t_{f}-t
$$

to the system of adjoint equations (8) and to the transversality conditions (9), we obtain the following left Riemann-Liouville fractional initial value problem (10)-(11):

$$
\left\{\begin{array}{l}
{ }_{0} D_{t^{\prime}}^{\alpha} p_{1}\left(t^{\prime}\right)=-\left[p_{1}\left(t^{\prime}\right)\left(\mu+\beta\left(t^{\prime}\right) I\left(t^{\prime}\right)\right)-\beta\left(t^{\prime}\right) I\left(t^{\prime}\right) p_{2}\left(t^{\prime}\right)\right], \\
{ }_{0} D_{t^{\prime}}^{\alpha} p_{2}\left(t^{\prime}\right)=-\left[p_{2}\left(t^{\prime}\right)(\mu+\varepsilon)-\varepsilon p_{3}\left(t^{\prime}\right)\right], \\
{ }_{0} D_{t^{\prime}}^{\alpha} p_{3}\left(t^{\prime}\right)=-\left[-\kappa_{1}+\beta\left(t^{\prime}\right) p_{1}\left(t^{\prime}\right) S\left(t^{\prime}\right)+p_{3}\left(t^{\prime}\right)\left(\mu+\nu+\mathbb{T}\left(t^{\prime}\right)\right)-p_{4}\left(t^{\prime}\right)\left(\nu+\mathbb{T}\left(t^{\prime}\right)\right)\right], \\
{ }_{0} D_{t^{\prime}}^{\alpha} p_{4}\left(t^{\prime}\right)=-\left[-\gamma p_{1}\left(t^{\prime}\right)+p_{4}\left(t^{\prime}\right)(\mu+\gamma)\right]
\end{array}\right.
$$

with initial conditions

$$
\left.p_{i}\left(t^{\prime}\right)\right|_{t^{\prime}=0}=0, \quad i=1, \ldots, 4 .
$$

Given the initial conditions (11), we solve (10) with the PECE procedure and obtain the values of the co-state variables $p_{i}, i=1, \ldots, 4$. The control is then updated by a convex combination of the previous control and the value from (77). This procedure is repeated iteratively until the values of the controls at the previous iteration are very close to the ones at the current iteration. To validate this algorithm, a fractional optimal control problem whose exact solution is known, Example 3.1 in [27, p. 86], was first successfully solved with it. Here we present our numerical results to the HRSV optimal control problem (3)-(6), for which an analytical solution is unknown.

Table 3: Initial conditions (5), in terms of percentage of total population, for the fractional optimal control problem (3) - (6) with parameters given by Table1, excepting angle $\Phi$ that is here assumed to be $\pi / 2$ so that the values correspond to the endemic equilibrium of (2).

\begin{tabular}{cccc}
\hline$S(0)$ & $E(0)$ & $I(0)$ & $R(0)$ \\
\hline 0,4081 & 0,0110 & 0,0278 & 0,5531 \\
\hline
\end{tabular}

In our numerical computations, we consider that $\mathbb{T}_{\max }=1$ and the other parameters are fixed according to Table 1. with exception of angle $\Phi$ that is assumed to be $\pi / 2$. Such value allows the transmission parameter initial value to be the average, $\beta(0)=b_{0}$, and the recruitment rate initial value to be also the average, $\lambda(0)=\mu$. Our initial conditions, given by Table 3 guarantee the existence of a non-trivial endemic equilibrium for the system (4) without control $(\mathbb{T}(t) \equiv 0)$, corresponding to the population system (2) prior introduction of treatment. Because World Health Organization goals for most diseases are usually fixed for five years periods, we assumed $t_{f}=5$. The solution to the fractional optimal control problem is displayed in Figures 3. 4(A) and 4(B) The periodic nature of the disease conditions the variation of the state variables. We can also see that the control is a continuous function with some non-regularity at the end of the time interval $\left[0, t_{f}\right]$. This behaviour is motivated by the irregular oscillation of the co-state variables, on which the control depends.

The intensity of treatment of the infectious individuals must have, periodically, in each year of the time interval, a given period of time during which most of the infectious individuals are 


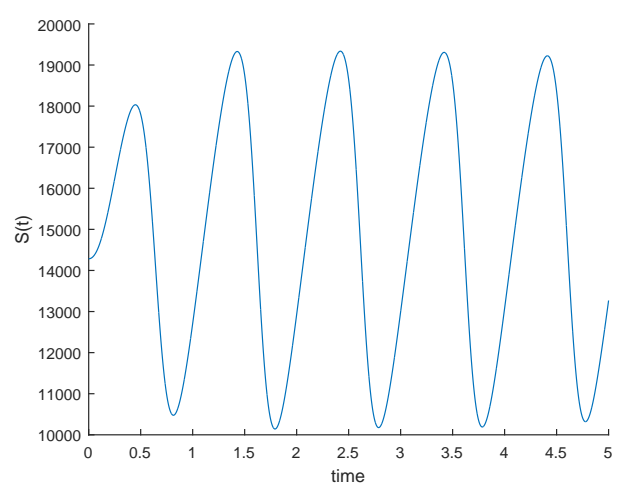

(A) Variation of the no. of susceptible individuals.

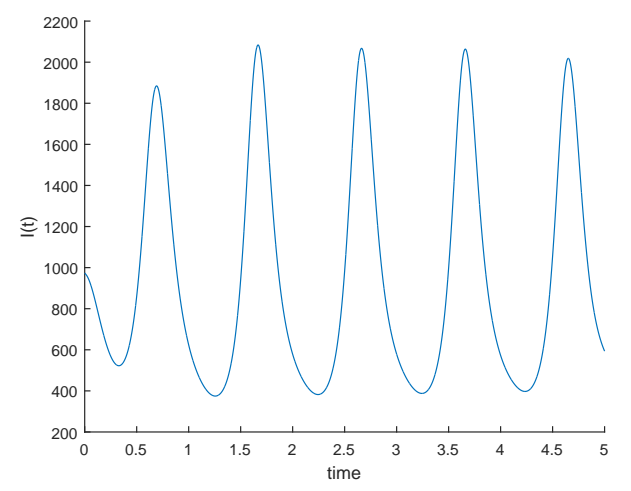

(C) Variation of the no. of infected individuals.

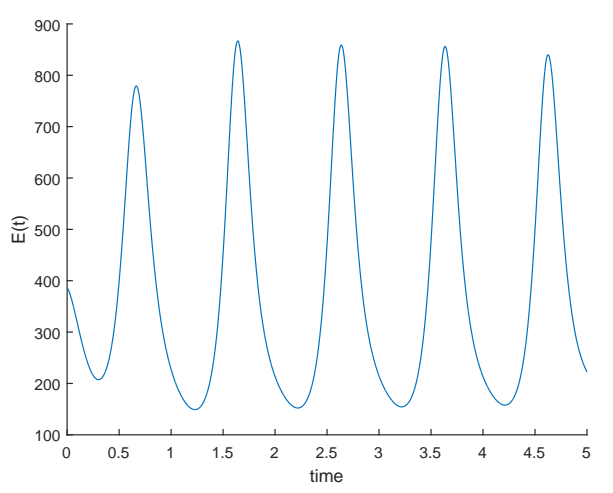

(B) Variation of the no. of exposed individuals.

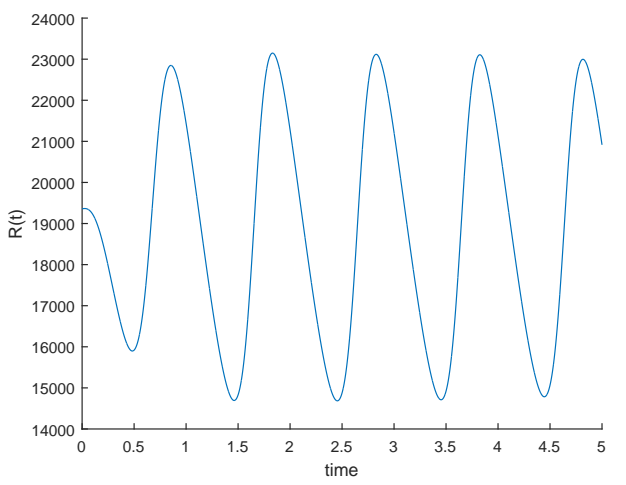

(D) Variation of the no. of recovered individuals.

Figure 3: State variables of the fractional optimal control problem (3) $-(6)$, considering $\alpha=0.993$ and weights $\kappa_{1}=1$ and $\kappa_{2}=0.001$.

treated. This ensures that the level of infectious reach very low levels. In Figure 4(C) the efficacy function [35] is exhibited. It is defined by

$$
F(t)=\frac{I(0)-I^{*}(t)}{I(0)}=1-\frac{I^{*}(t)}{I(0)},
$$

where $I^{*}(t)$ is the optimal solution associated with the fractional optimal control and $I(0)$ is the corresponding initial condition. This function measures the proportional variation in the number of infectious individuals after the application of the control $\mathbb{T}^{*}$, by comparing the number of infected individuals at time $t$ with the initial value $I(0)$ for which there is no control. We observe that $F(t)$ oscillates between -1.14 (lower bound) and +0.62 (upper bound), and exhibits the inverse tendency of $I(t)$.

Naturally, our results depend on the objective functional $\mathcal{J}$, defined in (3). They depend, namely, on the weight constants associated with the number of infectious individuals, $\kappa_{1}$, and with the cost of the treatment, $\kappa_{2}$. Figure 5 shows that the results do not change qualitatively by varying constants $\kappa_{i}, i=1,2$. However, the magnitude of the efficacy changes slightly when $\kappa_{1}$ and $\kappa_{2}$ vary independently.

To evaluate the cost and the effectiveness of the proposed fractional control measure during the intervention period, some summary measures are introduced. The total cases averted by the intervention during the time period $t_{f}$ is defined in [35] by

$$
A=t_{f} I(0)-\int_{0}^{t_{f}} I^{*}(t) d t
$$




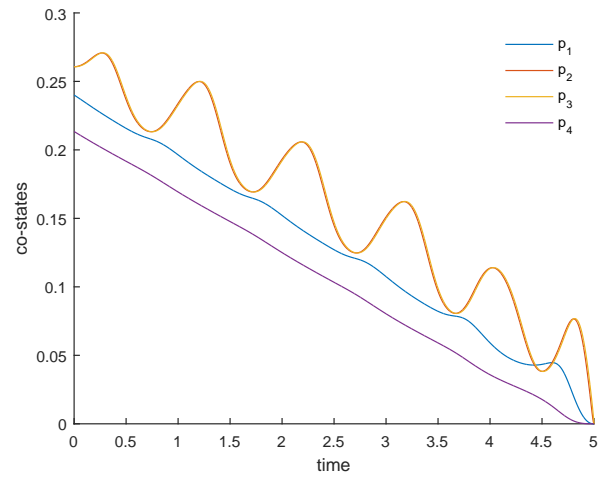

(A) Evolution of the four co-state variables.

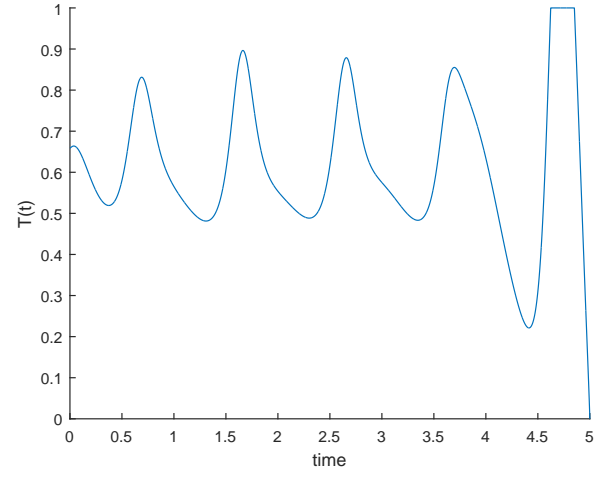

(B) Evolution of the optimal control $\mathbb{T}$ (treatment).

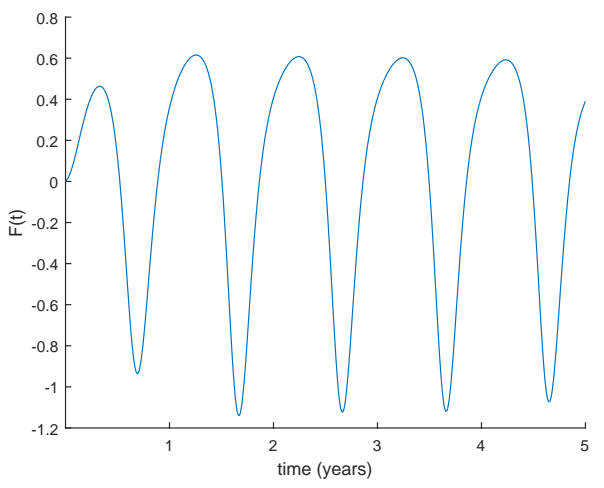

(C) Efficacy function $F(t)$ defined in (12).

Figure 4: Co-state variables $p_{i}, i=1, \ldots, 4$, extremal control (7) $\mathbb{T}$, and efficacy function (12) $F(t)$ associated to the fractional optimal control problem (3)-(6) with $\alpha=0.993$ and weights $\kappa_{1}=1$ and $\kappa_{2}=0.001$.

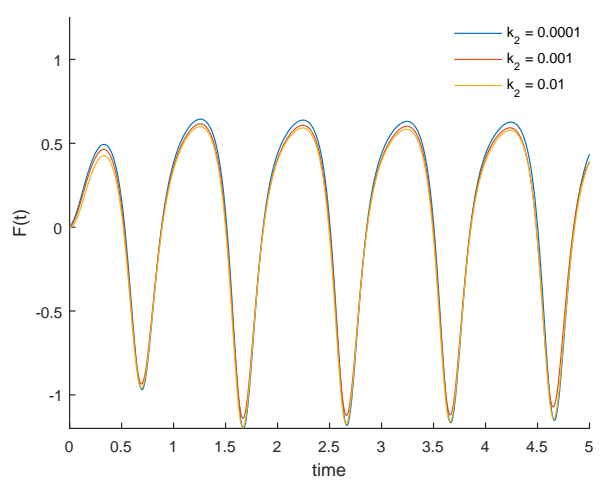

(A) $k_{1}=1$ and varying $k_{2}$.

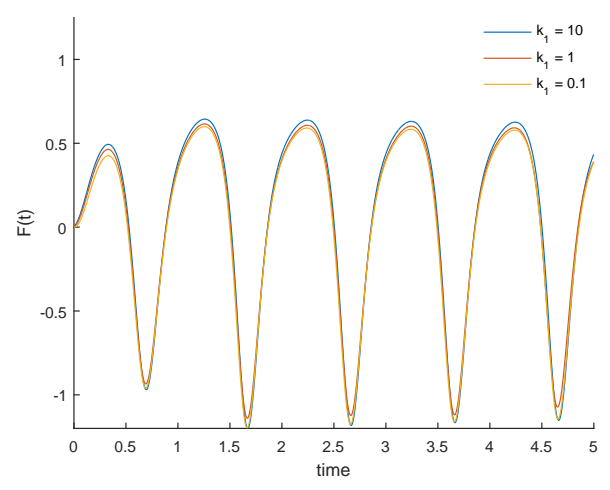

(B) $k_{2}=0.001$ and varying $k_{1}$.

Figure 5: Sensitivity analysis of the efficacy function $F(t)$ defined by (12) with respect to weights $\kappa_{1}$ and $\kappa_{2}$ of the objective functional (3). Left: $\kappa_{1}=1$ and $\kappa_{2}=0.01,0.001,0.0001$. Right: $\kappa_{2}=0.001$ and $\kappa_{1}=0.1,1,10$.

where $I^{*}(t)$ is the optimal solution associated with the fractional optimal control $\mathbb{T}^{*}$ and $I(0)$ is the corresponding initial condition. Note that this initial condition is obtained as the equilibrium 
proportion $\bar{I}$ of system (44) without treatment intervention, which is independent on time, so that $t_{f} I(0)=\int_{0}^{t_{f}} \bar{I} d t$ represents the total infectious cases over a given period of $t_{f}$ years. Let us define effectiveness as the proportion of cases averted on the total cases possible under no intervention [35]:

$$
\bar{F}=\frac{A}{t_{f} I(0)}=1-\frac{\int_{0}^{t_{f}} I^{*}(t) d t}{t_{f} I(0)} .
$$

The total cost associated with the intervention is defined in 35] by

$$
T C=\int_{0}^{t_{f}} C \mathbb{T}^{*}(t) I^{*}(t) d t
$$

where $C$ corresponds to the unit cost, per person, of detection and treatment of infectious individuals. Following [30, 35, the average cost-effectiveness ratio is given by

$$
A C E R=\frac{T C}{A}
$$

Table 4: Sumary of cost-effectiveness measures. Parameters according to Tables [1] and 3 with $\alpha=0.993$ and $C=1$.

\begin{tabular}{lccc}
\hline$A(13)$ & $T C(15)$ & $A C E R(16)$ & $\bar{F}(14)$ \\
\hline 172.7 & 3251.8 & 18.8 & 0.03547 \\
\hline
\end{tabular}

The cost-effectiveness measures for the fractional optimal control problem we have analyzed are summarized in Table 4. These results show limited effectiveness of the control treatment to reduce HRSV infectious individuals.

Another approach can be used to analyse the cost-effectiveness of the proposed fractional optimal control problem (3) - (6) and the classical optimal control $(\alpha=1)$ investigated in [36], by using the so-called incremental cost effectiveness ratio (ICER) [30]. This ratio is used to compare the differences between the costs and health outcomes of two alternative intervention strategies that compete for the same resources and it is generally described as the additional cost per additional health outcome. First, we rank the strategies in order of increasing effectiveness, here measured as the total infections averted $A$, defined in (13). Considering two contending strategies $a$ and $b$, the $I C E R$ of the strategy with the least effectiveness is its ACER and for the other strategies is given by

$$
\operatorname{ICER}(b)=\frac{T C(b)-T C(a)}{A(b)-A(a)}
$$

Table 5: Incremental cost-effectiveness ratio (17) for classical $(\alpha=1)$ and fractional $(\alpha=0.993)$ HRSV disease optimal control problems. Parameters according to Tables 1 and 3 with $C=1$.

\begin{tabular}{ccccc}
\hline$\alpha$ & $A(\underline{13})$ & $T C(\underline{15})$ & $A C E R(\underline{16})$ & $I C E R(17)$ \\
\hline 1.000 & 171.1 & 3242.1 & 18.9 & 18.9 \\
0.993 & 172.7 & 3251.8 & 18.8 & 6.06 \\
\hline
\end{tabular}

Results are shown in Table 5 . The fractional order strategy has the least $I C E R$ and therefore is more cost-effective than the classical strategy recently investigated in [36]. 


\section{Conclusion}

Human Respiratory Syncytial Virus (HRSV) is the most common cause of lower respiratory tract infection in infants and children worldwide. In addition, HRSV causes serious disease in elderly and immune compromised individuals. In this work, we discussed fractional compartmental models for HRSV. Estimation of the fractional order was performed for real data of Florida from September 2011 to July 2014, minimizing the $l_{2}$ norm. According to the obtained results, the proposed models fit well the real data. When we compare the optimal values for the fractional order SIRS- $\alpha$ and SEIRS- $\alpha$ models with the standard SIRS and SEIRS, one concludes that the absolute error of the fractional SIRS- $\alpha$ model reduces more than the homonym error of the fractional SEIRS$\alpha$ model. Thus, we can conclude that fractional derivatives give rise to theoretical models that allow a significant improvement in the fitting of real data, when compared with analogous classical models, particularly in simpler cases. However, our results on fractional optimal control show that treatment has a limited effect on HRSV infected individuals. Nevertheless, a cost-effectiveness analysis of the proposed fractional order strategy shows that it is more cost-effective than the classical strategy followed in the literature. As future work, we plan to investigate the usefulness of our fractional approach in other geographical regions.

\section{Acknowledgements}

Rosa was supported by the Portuguese Foundation for Science and Technology (FCT) through IT (project UID/EEA/50008/2013); Torres by FCT through CIDMA (project UID/MAT/04106/2013) and TOCCATA (project PTDC/EEI-AUT/2933/2014 funded by FEDER and COMPETE 2020). The authors are very grateful to the comments and suggestions from two anonymous reviewers, which helped them to enrich the paper.

\section{References}

[1] Acedo L, Moraño J-A, Díez-Domingo J. Cost analysis of a vaccination strategy for respiratory syncytial virus (RSV) in a network model. Math. Comput. Modelling 2010;52(7-8):1016-1022.

[2] Agarwal P, Al-Mdallal Q, Cho YJ, Jain S. Fractional differential equations for the generalized Mittag-Leffler function. Adv. Difference Equ. 2018; Paper no. 58, 8 pp.

[3] Agarwal P, Choi J, Paris RB. Extended Riemann-Liouville fractional derivative operator and its applications. J. Nonlinear Sci. Appl. 2015;8(5):451-466.

[4] Agarwal P, El-Sayed AA. Non-standard finite difference and Chebyshev collocation methods for solving fractional diffusion equation. Phys. A 500 2018: 40-49.

[5] Agarwal P, Jain S, Mansour T. Further extended Caputo fractional derivative operator and its applications. Russ. J. Math. Phys. 2017;24(4):415-425.

[6] Agarwal P, Nieto JJ, Luo MJ. Extended Riemann-Liouville type fractional derivative operator with applications. Open Math. 2017;15:1667-1681.

[7] Ali HM, Lobo Pereira F, Gama, SMA. A new approach to the Pontryagin maximum principle for nonlinear fractional optimal control problems. Math. Methods Appl. Sci. 2016;39(13):3640-3649.

[8] Almeida R, Pooseh S, Torres DFM. Computational methods in the fractional calculus of variations. London: Imperial College Press; 2015.

[9] Almeida R, Tavares D, Torres DFM. The variable-order fractional calculus of variations. SpringerBriefs in Applied Sciences and Technology. Springer, Cham, 2019. arXiv:1805.00720 
[10] Area I, Losada J, Ndaïrou F, Nieto JJ, Tcheutia DD. Mathematical modeling of 2014 Ebola outbreak. Math. Methods Appl. Sci. 2017;40(17):6114-6122.

[11] Area I, Ndaïrou F, Nieto JJ, Silva CJ, Torres DFM. Ebola model and optimal control with vaccination constraints. J. Ind. Manag. Optim. 2018;14(2):427-446. arXiv:1703.01368

[12] Arenas AJ, González G, Jódar L. Existence of periodic solutions in a model of respiratory syncytial virus RSV. J. Math. Anal. Appl. 2008;344(2):969-980.

[13] Arenas AJ, Moraño JA, Cortés, JC. Non-standard numerical method for a mathematical model of RSV epidemiological transmission. Comput. Math. Appl. 2008;56(3):670-678.

[14] Bastos NRO, Mozyrska D, Torres DFM. Fractional derivatives and integrals on time scales via the inverse generalized Laplace transform. Int. J. Math. Comput. 2011;11(J11):1-9. arXiv:1012.1555

[15] Bayour B, Torres DFM. Complex-valued fractional derivatives on time scales. Differential and difference equations with applications, 79-87, Springer Proc. Math. Stat., 164, Springer, [Cham], 2016. arXiv:1511.02153

[16] Carvalho ARM, Pinto CMA, Baleanu D. HIV/HCV coinfection model: a fractional-order perspective for the effect of the HIV viral load. Adv. Difference Equ. 2018; Paper no. 2, $22 \mathrm{pp}$.

[17] Çetinkaya A, Kıymaz İ; Agarwal P, Agarwal R. A comparative study on generating function relations for generalized hypergeometric functions via generalized fractional operators. Adv. Difference Equ. 2018; Paper no. 156, 11 pp.

[18] Choi J, Agarwal P, Jain S. Certain fractional integral operators and extended generalized Gauss hypergeometric functions. Kyungpook Math. J. 2015;55(3):695-703.

[19] Debbouche A, Nieto JJ, Torres DFM. Optimal solutions to relaxation in multiple control problems of Sobolev type with nonlocal nonlinear fractional differential equations. J. Optim. Theory Appl. 2017;174(1):7-31. arXiv:1504.05153

[20] Diethelm K, Ford NJ, Freed AD, Luchko Yu. Algorithms for the fractional calculus: a selection of numerical methods. Comput. Methods Appl. Mech. Engrg. 2005;194(6-8):743-773.

[21] Florida Department of Health. Respiratory Syncytial Virus. Tallahassee, Florida (FL), USA. Available from: http://www.floridahealth.gov/diseases-and-conditions/respiratory-syncytial-virus Accessed: May 3, 2017.

[22] Glezen WP, Taber LH, Frank AL, Kasel JA. Risk of primary infection and reinfection with respiratory syncytial virus. Amer. J. Dis. Child. 1986;140(6):543-546.

[23] Hall CB, Weinberg GA, Iwane MK, et al. The burden of respiratory syncytial virus infection in young children. New Engl. J. Medic. 2009;360(6):588-598.

[24] Jahanshahi S, Torres DFM. A simple accurate method for solving fractional variational and optimal control problems. J. Optim. Theory Appl. 2017;174(1):156-175. arXiv:1601.06416

[25] Kamocki R. Pontryagin maximum principle for fractional ordinary optimal control problems. Math. Methods Appl. Sci. 2014;37(11):1668-1686.

[26] Kıymaz İO, Agarwal P, Jain S, Çetinkaya A. On a new extension of Caputo fractional derivative operator. Advances in real and complex analysis with applications, 261-275, Trends Math., Birkhäuser/Springer, Singapore, 2017.

[27] Malinowska AB, Torres DFM. Introduction to the fractional calculus of variations. London: Imperial College Press; 2012. 
[28] Mateus J, Rebelo P, Rosa S, Silva C, Torres DFM. Optimal control of non-autonomous SEIRS models with vaccination and treatment. Discrete Contin. Dyn. Syst. Ser. S 2018;11(6):11791199. arXiv: 1706.06843

[29] Ndaïrou F, Area I, Nieto JJ, Silva CJ, Torres DFM. Mathematical modeling of Zika disease in pregnant women and newborns with microcephaly in Brazil. Math. Methods Appl. Sci., in press. DOI:10.1002/mma.4702 arXiv:1711.05630

[30] Okosun KO, Rachid O, Nizar Marcus. Optimal control strategies and cost-effectiveness analysis of a malaria model. Biosystems 2013;111(2):83-101.

[31] Podlubny I. Fractional differential equations. San Diego, CA: Academic Press, Inc.; 1999.

[32] Pooseh S, Rodrigues HS, Torres DFM. Fractional derivatives in dengue epidemics. AIP Conf. Proc. 2011; 1389(1):739-742. arXiv:1108.1683

[33] Rachah A, Torres DFM. Predicting and controlling the Ebola infection. Math. Methods Appl. Sci. 2017;40(17):6155-6164. arXiv:1511.06323

[34] Rachah A, Torres DFM. Analysis, simulation and optimal control of a SEIR model for Ebola virus with demographic effects. Commun. Fac. Sci. Univ. Ank. Sér. A1 Math. Stat. 2018;67(1):179-197. arXiv:1705.01079

[35] Rodrigues P, Silva CJ, Torres DFM. Cost-effectiveness analysis of optimal control measures for tuberculosis. Bull. Math. Biol. 2014;76(10):2627-2645. arXiv:1409.3496

[36] Rosa S, Torres DFM. Parameter estimation, sensitivity analysis and optimal control of a periodic epidemic model with application to HRSV in Florida. Stat. Optim. Inf. Comput. 2018;6(1):139-149. arXiv:1801.09634

[37] Ruzhansky M, Cho YJ, Agarwal P, Area I. Advances in real and complex analysis with applications, Trends in Mathematics, Birkhäuser/Springer, Singapore, 2017.

[38] Saoudi K, Agarwal P, Kumam P, Ghanmi A, Thounthong P. The Nehari manifold for a boundary value problem involving Riemann-Liouville fractional derivative. Adv. Difference Equ. 2018; Paper no. 263, 18 pp.

[39] Sidi Ammi MR, Jamiai I, Torres DFM. Global existence of solutions for a fractional Caputo nonlocal thermistor problem. Adv. Difference Equ. 2017; Paper no. 363, 14 pp. arXiv:1711.00143

[40] Tavares D, Almeida R, Torres DFM. Combined fractional variational problems of variable order and some computational aspects. J. Comput. Appl. Math. 2018;339:374-388. arXiv: 1704.06486

[41] Weber A, Weber M, Milligan P. Modeling epidemics caused by respiratory syncytial virus (RSV). Math. Biosci. 2001;172(2):95-113.

[42] Wojtak W, Silva CJ, Torres DFM. Uniform asymptotic stability of a fractional tuberculosis model. Math. Model. Nat. Phenom. 2018;13(1):Art. 9, 10 pp. arXiv:1801.07059

[43] Zafar ZUA Rehan K, Mushtaq M. HIV/AIDS epidemic fractional-order model. J. Difference Equ. Appl. 2017;23(7):1298-1315.

[44] Zhang T, Liu J, Teng Z. Existence of positive periodic solutions of an SEIR model with periodic coefficients. Appl. Math. 2012;57(6):601-616. 